\title{
III-Nitride Semiconductors based Optical Power Splitter Device Design for underwater Application
}

\author{
Retno Wigajatri Purnamaningsih ${ }^{1}$, Nyi Raden Poespawati ${ }^{2}$, Elhadj Dogheche ${ }^{3}$ \\ ${ }^{1,2}$ Department of Electrical Engieering, Universitas Indonesia, Indonesia \\ ${ }^{3}$ DOAE Optoelectronics Group, Valenciennes University, France
}

\begin{tabular}{l} 
Article Info \\
\hline Article history: \\
Received May 26, 2018 \\
Revised Aug 2, 2018 \\
Accepted Aug 9, 2018 \\
\hline
\end{tabular}

Keyword:

FD-BPM method

III-nitrides

MMI structure

Optical power splitter

Taper waveguide

\begin{abstract}
In this paper, we introduce III-nitrides based $1 \times 4$ optical power splitter for underwater optical communication applications. To the best of our knowledge, this is a first study for the design of multimode interference (MMI) and four-branch taper waveguide based on GaN/sapphire. The microstructure of $\mathrm{GaN}$ semiconductor grown by Metalorganic Chemical Vapor Deposition (MOCVD) on (0001) sapphire reported. The numerical experimental is conducted using the 3D FD-BPM method. The results showed that the optical power splitter has an excess loss of $0.013 \mathrm{~dB}$ and imbalance of $0.17 \mathrm{~dB}$. The results open the opportunity for the future device using this technology for the underwater application.
\end{abstract}

Copyright $\left({ }_{0} 2018\right.$ Institute of Advanced Engineering and Science. All rights reserved.

\section{Corresponding Author:}

Retno Wigajatri Purnamaningsih, Departement of Electrical Engineering, Universitas Indonesia, Kampus UI Depok, Depok, Indonesia. Email: retno.wigajatri@ui.ac.id

\section{INTRODUCTION}

Underwater telecommunication has attracted many scientists since it plays an important role such as in military, surveillance, offshore surveying, and oceanography research. In order to facilitate these activities, unmanned vehicles or robot have been operated underwater [1]. These activities require high bandwidth and high capacity for information transfer in underwater. So far acoustic communication is the most commonly used in an underwater communication system with ranges of several kilometers. However acoustic communication is extremely slow (only kilobits per second) with high latency caused by reflections, and relatively slow speed of sound underwater [2]. Moreover, the effect of underwater acoustic noise strongly depends on the diurnal variability of waters [3]. Therefore, it is not possible to be applied for transmitting high data rates in real-time such as video.

Optical communication became a choice to overcome that problem. The biggest challenge for underwater communication is the fundamental characteristic of ocean seawater. The optical attenuation characteristic of deep sea water has a low absorption roughly between 400 and $500 \mathrm{~nm}$ in the blue-green range of the visible spectrum [3], [4]. Therefore to minimize the required power for marine applications, the optical source should emit in this ocean window. Research on underwater wireless optical communications (UWOC) using Gallium nitride (GaN) blue laser diode (LD) based free-space visible light communication (VLC) had been presented [5]. Experimental research employing 450-nm source and a fiber-pigtailed laser diode (LD) had successfully achieved over short transmission distance [6]. High-speed UOWC using a blueemitting GaN-based micro-LED for short distance has been reached [7]. A high-speed air-water optical wireless communication system for both downlink and uplink transmission using pigtailed green-light laser 
diode (LD) has also been experimentally demonstrated [8]. Study on performance analysis of various modulation techniques for free space optical communication system has also been conducted [9].

The achievements were due to the advancement of high-powered blue, green lasers, LED and photodetector [10]-[12] using III-nitrides semiconductors. Besides their abilities to emit and detect in UVblue region, this semiconductor has promising application in passive and active optical waveguide based devices.

Various design of photonic devices have been developed, among others are waveguide intersection, coupler, demultiplexer, and optical power splitter [13]-[17]. Optical power splitters are one of the most elementary devices in a photonic system. The quality of splitter plays an important role to achieve the system performance. Recently, researchers have demonstrated splitter for $\mathrm{C}$ band range employed seven horizontal III-nitride slotted waveguide surrounded by silicon [18].

III-nitrides are prominent for its superior electrical properties, its temperature stability and its mechanical hardness, which makes it suitable for the harsh environment [19]. Gallium nitride with Aluminum nitride (AIN) buffer layer shows the ability to integrate on sapphire substrates with small propagation loss [20].

The design of III-nitride based on Y junction, MMI structure splitter used for high-speed optical communication systems have been demonstrated [21], [22]. Design of free standing GaN waveguides has also reported [23]. Recently a photonic integration for multifunctional devices based on this material has been developed [24]. However, to our knowledge, the application of photonic waveguide using III-Nitrides materials for underwater communication has not yet been explored. In this paper, for the first time, we present the design of a $1 \times 4$ optical power splitter using gallium nitride $(\mathrm{GaN})$ on sapphire for the underwater application. The light is propagating in the splitter based on beam propagation method. Numerical optimizations were conducted on the sizes of input and output waveguides and the intermediate multimode interference structure (MMI) in order to obtain an efficient splitting of the transverse electric (TE) mode.

In order to achieve high-performance device, high-quality microstructures are required. Therefore the Microstructure of GaN semiconductor grown by Metalorganic Chemical Vapor Deposition (MOCVD) on (0001) sapphire is also reported.

\section{GaN MATERIALS MICROSTRUCTURE}

The III-nitrides crystallize into two crystallographic structures: the cubic zinc-blende and the hexagonal wurtzite structures. The wurtzite structures are commonly studied since it is thermodynamically stable under ambient conditions [20]. The III-nitride system includes three binary compounds consisting of aluminum nitride $(\mathrm{AlN})$, gallium nitride $(\mathrm{GaN})$, and indium nitride $(\mathrm{InN})$; three ternary compounds of AlxGa1-xN, InxGa1-xN, and InxAl1-xN; and the quaternary compounds AlxInyGa1-x-yN. This system is particularly suitable for Optoelectronics since its band gap continuously ranges from $6.2 \mathrm{eV}$ for AlN to $1 \mathrm{eV}$ for InN, which covers the entire visible, near ultraviolet and near-infrared portions of the electromagnetic wave spectrum [21].

In this paper, the GaN materials used for optical waveguide design are grown by Metalorganic Chemical Vapor Deposition (MOCVD) on (0001) sapphire using an AlN/GaN Short Period Superlattice (SPS).Trimethylaluminum and Trimethylgallium used as alkyl sources and ammonia used as a hydride source. After the cleaning of the substrate at high temperature and the growth of a thin AlN buffer layer (150 nm layer thickness), low temperature (LT) $\mathrm{AlN}$ is grown at $950^{\circ} \mathrm{C}$. The temperature elevated to $1040^{\circ} \mathrm{C}$ and the deposition of a $150 \mathrm{~nm}$ thick high temperature (HT) grown on plane AlN. Finally, an interlayer consisting of 10 times GaN/AlN layers having a total thickness of $200 \mathrm{~nm}$ were carried out under the same HT conditions, and $\mathrm{GaN}$ has grown on top of the interlayer. The experimental process is described in the literature [21].

The microstructure mainly characterized by the crystallinity of the layers, homogeneity, and dislocation density. To study the microstructural defects existing in the layers, TEM analysis (Tecnai G2-20) conducted at UMET-CNRS (Unité Matériaux et Transformations). Figure 1 shows a large scale crosssectional weak-beam view of the layers, consist of the sapphire substrate, AlN/GaN SPS buffer layer, and $\mathrm{GaN}$ top layer. The images were taken along $\mathrm{g}=0002$ diffraction vector. Few threading dislocations observed through the GaN layer. 


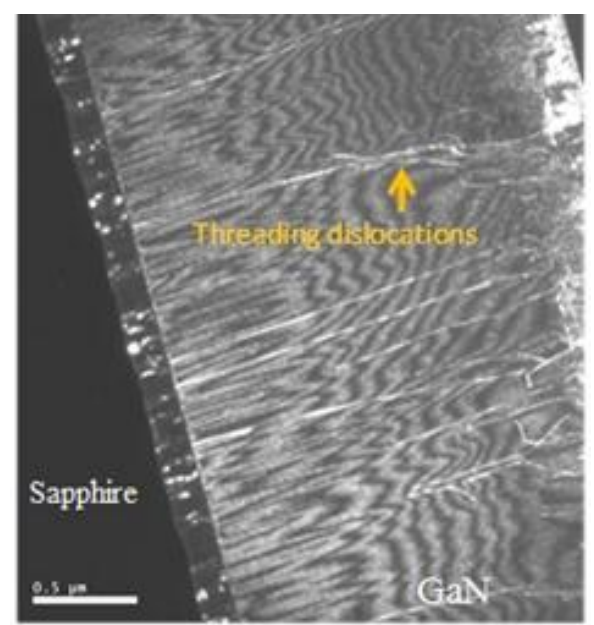

Figure 1. TEM image of GaN on sapphire substrate with AlN/GaN SPS as the buffer layer

\section{PROPOSED DESIGN AND THEORETICAL ASPECT OF GAN-BASED OPTICAL POWER SPLITTER}

In this paper, we proposed a $1 \times 4$ optical power splitter using rib waveguide, a rectangular MMI structure, and four taper waveguides. The MMI structure was chosen due to their excellent properties and easy to fabricate [25]. The use of taper waveguide has been selected to improve the performance of the splitter. Figure 2 shows the proposed design configuration of the splitter. $L_{M M I}, W_{M M I}$ are the length and the width of MMI structure. In this design, the height $(H)$ of all the structure is the same. Figure 3 presents the schematic illustration of $\mathrm{GaN}$ structure layer in the $\mathrm{x}-\mathrm{y}$ plane.

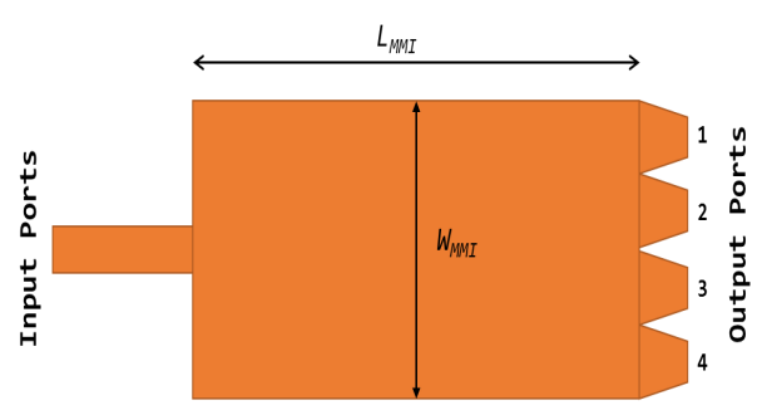

Figure 2. The schematic structure of the proposed four branches optical power splitter in $x-z$ plane

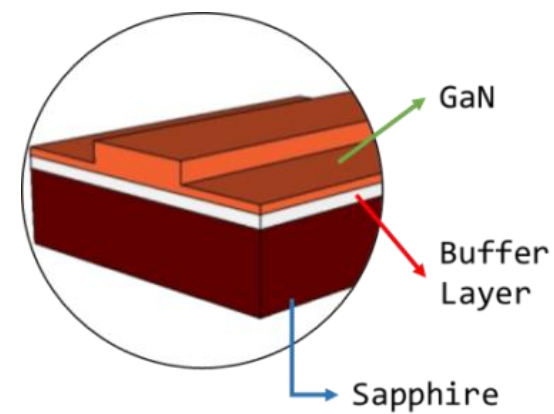

Figure 3. The schematic ilustration of GaN structure layer in $x-y$ plane

As a cladding material, we used air (no color) with refractive index $n_{c l}$, sapphire (maroon color) used as the wafer $\left(n_{\text {wafer }}\right), \mathrm{AIN}$ and $\mathrm{AlN} / \mathrm{GaN}$ (white color) as the buffer layer, and GaN (dark orange color) used as the waveguide material $\left(n_{\text {core }}\right)$. The refractive indices of cladding and wafer $\left(n_{c l}, n_{w a f e r}\right)$ at $0.45 \mu \mathrm{m}$ are 1.00 and 1.76 respectively, while the effective refractive index of GaN layer for TE mode is 2.279. This value obtained through prism coupling measurement. In this design, we used the wurtzite structures sapphire due to its temperature stability, which can increase the performances of the splitter.

MMI structure works according to self-imaging principle, where the input field profile is reproduced in single or multiple images along the propagation direction of the waveguide at periodic intervals [25]. The basic idea of the design is to obtain mode interference between the fundamental and the higher order mode propagated in the multimode section, high power confinement, and uniform optical power. The length of MMI structure, $L_{M M I}$ can be predicted by [26]:

$$
L_{M M I}=\frac{3 p L_{\pi}}{4 N}
$$


Here $L_{\pi}$ is the beat length of the two lowest order mode

$$
L_{\pi} \cong \frac{4 n_{\text {core }} W_{e}^{2}}{3 \lambda}
$$

Where $\mathrm{N}$ is the number of the output port, $W_{e}$ is the effective width of MMI region, $n_{\text {core }}$ is the effective refractive index of $\mathrm{GaN}$.

Equation (1) and Equation (2) can be used to predict the $L_{M M I}$ value. The other MMI parameters are chosen such that the MMI section supports four modes propagation. The input rib waveguide centered with the MMI section. To achieve the desired interference, the length of MMI section should be optimized based on the predicted results. For a step-index multimode waveguide of width $W_{M M I}$ are influenced by light polarization and lateral penetration depth of each mode field, associated with the Goos-Hanchen shifts, therefore $W_{M M I}$ will be slightly altered to $W_{\text {eff }}$ according to Equation (3).

$$
W_{e}=W_{M M I}+\left(\frac{\lambda}{\pi}\right)\left(\frac{n_{c l}}{n_{\text {core }}}\right)^{2 \sigma}\left(n_{\text {core }}^{2}-n_{c l}^{2}\right)^{-(1 / 2)}
$$

Where $n_{c l}$ is cladding refractive index

In this work, the refractive index difference between core and cladding is high. Therefore the penetration depth is very small so that the effective width of the MMI coupler, $W_{e f f}$, and the operating wavelength $\lambda$ formulated as in Equation (4). For TE mode [26]:

$$
W_{e f f}=W_{M M I}+\lambda_{0} / \pi\left(n_{c l a d}^{2}-n_{e f f}^{2}\right)^{-1 / 2}
$$

To describe self-imaging phenomena in multimode waveguides, the finite difference beam propagating method is one of the most powerful techniques. This method can be used to investigate both linear and nonlinear light wave propagation phenomena in axially varying waveguides [25], [26]. To solve Maxwell's equations, we used finite difference BPM, in place of partial derivatives. It is computationally intensive. Besides, provides the basis for numerical modeling and design, BPM is also presented the interference mechanism in the multimode structure [26], [27].

The numerical experimental is conducted using OptiBPM software based on a slowly varying envelope approximation) combined with Matlab software. The field propagation calculation through the structure is carried out using the finite difference beam propagation method (FD-BPM). This approach is chosen since it can automatically include the calculation of all the guided and non-guided modes. The numerical experiment is carried out for underwater optical telecommunication. In order to support the fundamental TE mode and confine the light inside the GaN layer, the numerical experiments conducted in stages. First, we optimize the width $\left(W_{M M I}\right)$ and the length $\left(L_{M M I}\right)$ of MMI structure to obtain four separate modes at the output side. Then, the four taper branches are integrated to MMI structure to construct the optical power splitter.

\section{SIMULATION RESULTS AND DISCUSSION}

After computer experiment using 3D BPM to optimize the width and length of the rib waveguide, MMI and taper structure, we obtained the optimum design of four branch power splitter at the $\mathrm{x}-\mathrm{y}$ plane. The influence of the waveguide width is also analyzed. The waveguide width of $4 \mu \mathrm{m}$ chosen in order to ensure that only the fundamental TE mode propagates in the device. From the numerical experiment, it found that best distance between the center of two adjacent output waveguide is $6 \mu \mathrm{m}$. The distance is needed to avoid significant coupling between the output waveguides. It can also be noticed that the optimal width values of the output waveguide taper are varied from 6-4 $\mu \mathrm{m}$. In order to obtain the best power output distribution, the length of the taper structure needs to be optimized. Figure 4 indicates that all the output ports almost have the same amplitude at $100 \mu \mathrm{m}$. Below and above this length the power output the structure is not uniform. This implies that the separation between the adjacent waveguides is sufficiently close to enable some electromagnetic energy transferred to the neighboring guide.

Final simulation results show that the best result for TE polarization achieved when the length and the width of power splitter are $1350 \mu \mathrm{m}$ and $25 \mu \mathrm{m}$ respectively, with $25 \mu \mathrm{m}$ width and $950 \mu \mathrm{m}$ length of MMI structure. This result gives the minimum imbalance and lowest excess loss at $0.45 \mu \mathrm{m}$. 


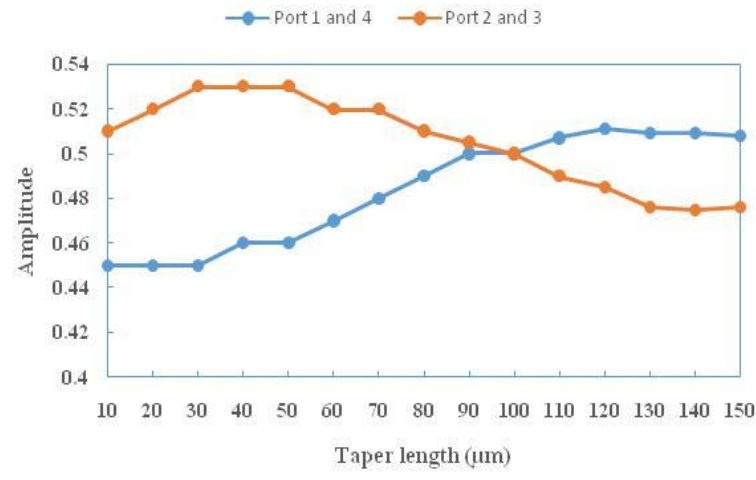

Figure 4. Relatif amplitude distribution of four taper output waveguide for various taper length

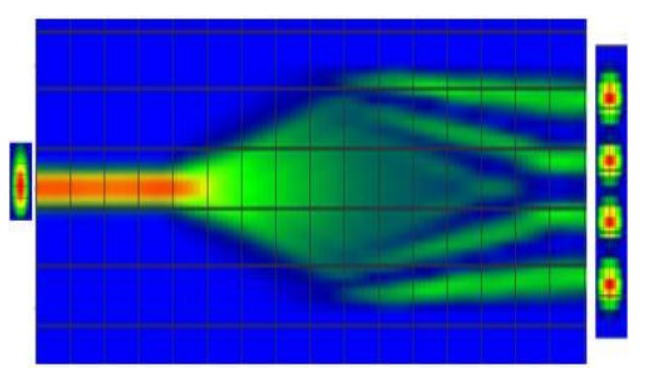

Figure 5. Optical intensity field distribution of four branch power splitter along propagation distance with total length and width $1350 \mu \mathrm{m}$ and $25 \mu \mathrm{m}$ respectively

Figure 5 presents the map of optical field distribution along the structure. Red, yellow and green color represents the level of the optical field. It shows that a strong confinement (red color) obtain inside the $\mathrm{GaN}$ layer. It is also shown that the optical power split almost equally at $\mathrm{z}=1250 \mu \mathrm{m}$ and divided into four ports at the output of the proposed device.

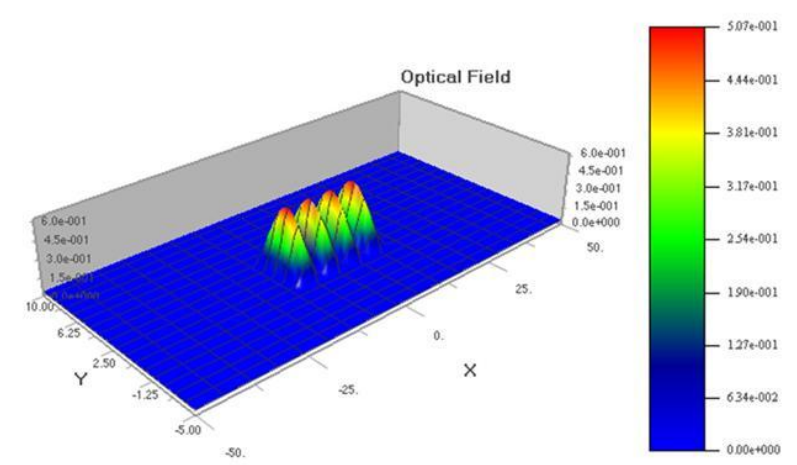

Figure 6. 3D optical intensity field distribution at the output ports

Figure 6 shows the optical intensity field distribution at the power splitter output. The proposed design achieves an almost perfect splitting ratio of $25 \%, 24.9 \%, 24.9 \%$ and $25 \%$.

To anticipate the fabrication error, we investigate the tolerance value of the width of MMI structure.. The results are shown in Figure 7.

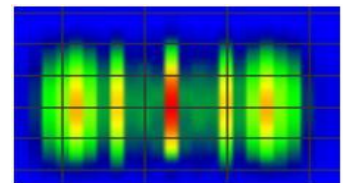

(a)

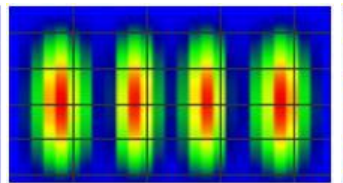

(b)

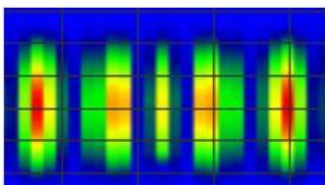

(c)

Figure 7. Optical fields profile at the output ports for various width (a) $24.5 \mu \mathrm{m}$ (b) $25 \mu \mathrm{m}$ and (c) $25.5 \mu \mathrm{m}$ with MMI length of $950 \mu \mathrm{m}$. 
Figure 7 shows that the tolerance fabrication for MMI width should be maintained under $0.5 \mu \mathrm{m}$ from the optimum width of $25 \mu \mathrm{m}$, above this value, the unsymmetric optical field distribution will appear at the output port. This is because the self imaging effect caused by multimode interference phenomena reproduced only at certain periodic interval of optical fields propagation in MMI structure.

In order to obtain the best design, GaN thicknes is also analyzed. Figure 8 shows the optical fields profile at the output ports for various GaN thickness. It can be noticed that in the thickness range of 1 up to 4 $\mu \mathrm{m}$, the proposed structure supports fundamental quasi TE mode along the output branch. In addition, the influence of thickness of $\mathrm{GaN}$ is analyzed by taking into account the propagation losses along the structure and fabrication process. It can be noticed from Figure 9 that the optical power output relatively stable for $\mathrm{GaN}$ thickness range of 1.5 up to $4 \mu \mathrm{m}$. Therefore the preffered thickness of $1.5 \mu \mathrm{m}$ has been chosen for the design, at this GaN thickness, the relative transmitted optical power is 0.997 .

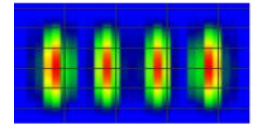

(a)

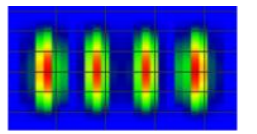

(b)

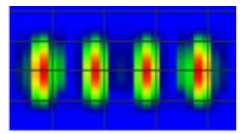

(c)

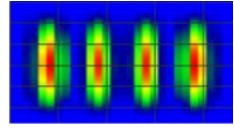

(d)

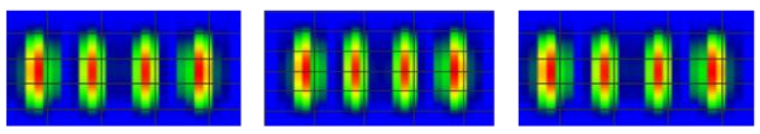

(e)

(f)

(g)

Figure 8. Optical mode profile at the output ports for various GaN layer thickness: $1 \mu \mathrm{m}$ (a) $1.5 \mu \mathrm{m}$ (b) $2 \mu \mathrm{m}$ (c) $2.5 \mu \mathrm{m}$ (d) $3 \mu \mathrm{m}$ (e) $3.5 \mu \mathrm{m}$ (f) $4 \mu \mathrm{m}$ (g)

The relative optical power as a function of propagation distance of the proposed splitter at $\lambda=0.45 \mu \mathrm{m}$ displayed in Figure 10. Red line indicated the value of relative optical power along propagation distance.

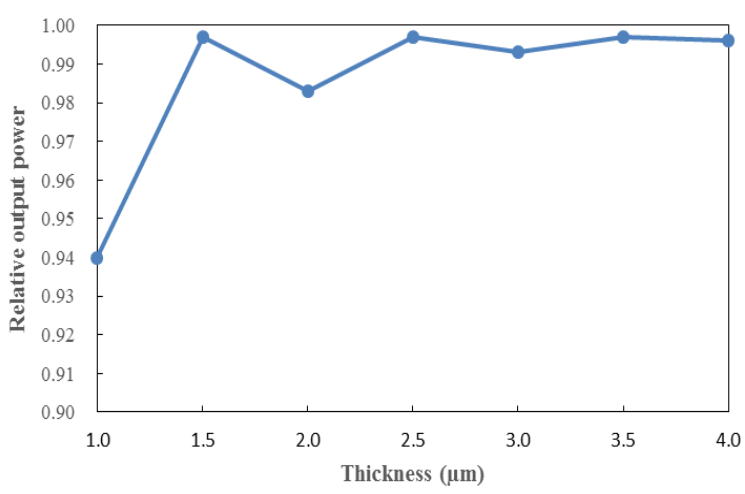

Figure 9. Relative optical output power for various GaN layer thickness

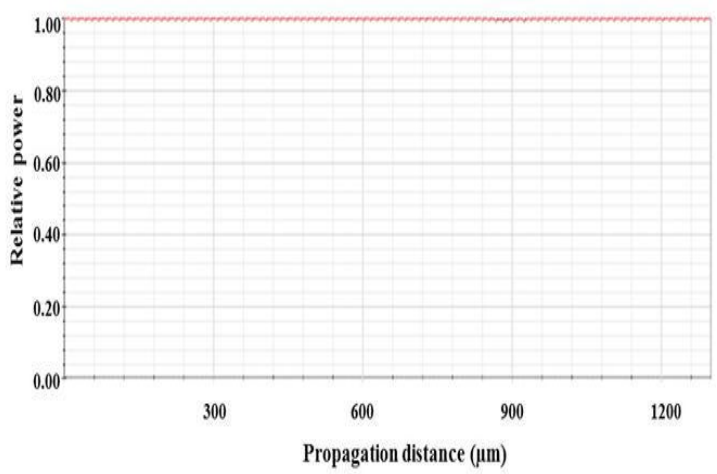

Figure 10. The total relative power along the power splitter at $\lambda=0.45 \mu \mathrm{m}$ 
Figure 10 shows that the total relative power along the power splitter is stable with the value of 0.997 at the output ports. The influence of the light wavelength is also investigated for the range of 0.4 up to $0.5 \mu \mathrm{m}$. The result is shown in Figure 11 .

However since laser input has a spectrum width in nanometers, the simulation is undertaken for $\lambda=0.4-0.5 \mu \mathrm{m}$. The normalized transmitted power varies from 0.997 to 0.940 over $100 \mathrm{~nm}$ spectrum scope as shown in Figure 11. It is found that the design relatively sensitive to wavelength variation and shows that the maximum relative output power obtained at $\lambda=0.45 \mu \mathrm{m}$. This phenomena is caused by the interference effect in guiding mechanism along MMI structure.

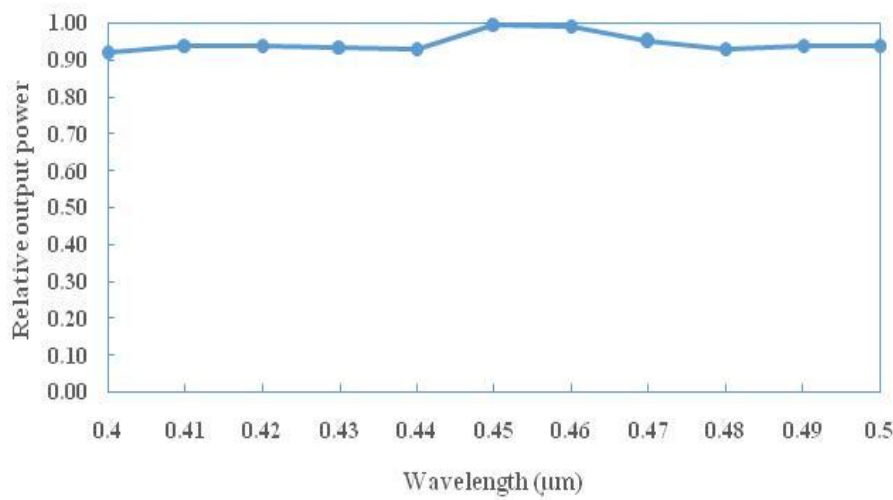

Figure 11. Relative power distribution for wavelength range from 0.4 up to $0.5 \mu \mathrm{m}$

The key performance of power splitters is excess loss and imbalance. Therefore we calculate both excess loss and imbalance for the optimum width and length to obtain the lowest loss. The excess loss and power imbalance of the splitter defined as in Equations (5) and (6) [18]:

$$
\begin{aligned}
& \text { Excess Loss }=-10 \log _{10}\left(P_{\text {out }} / P_{\text {in }}\right) \\
& \text { Imbalance }=10 \log _{10}\left(P_{\text {outmin }} / P_{\text {outmax }}\right)
\end{aligned}
$$

Where $P_{\text {out }}$ is the total optical output power, and $P_{\text {in }}$ is the optical powers coupled into the input waveguide, while $P_{\text {outmin }}$ and $P_{\text {outmax }}$ are the lowest and highest optical power at the output ports. From the calculation, we obtained that the power splitter has an excess loss of $0.013 \mathrm{~dB}$ as shown in Figure 12 and yields a maximum power imbalance of $0.17 \mathrm{~dB}$ at $\lambda=450 \mathrm{~nm}$ (Figure 13). Compared to other design of $1 \times 4$ optical power splitter using Silicon on insulator (SOI) for C band-range [18], our design exhibits a higher imbalance but lower excess loss.

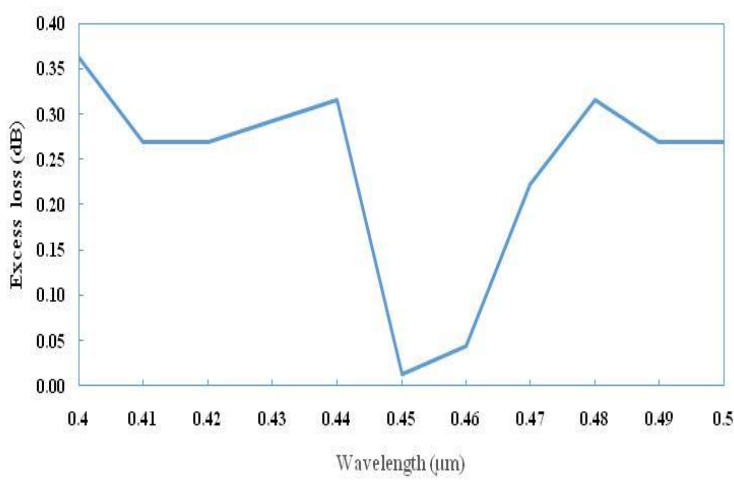

Figure 12. Excess loss of the TE mode in the proposed design $1 \times 4$ optical power splitter

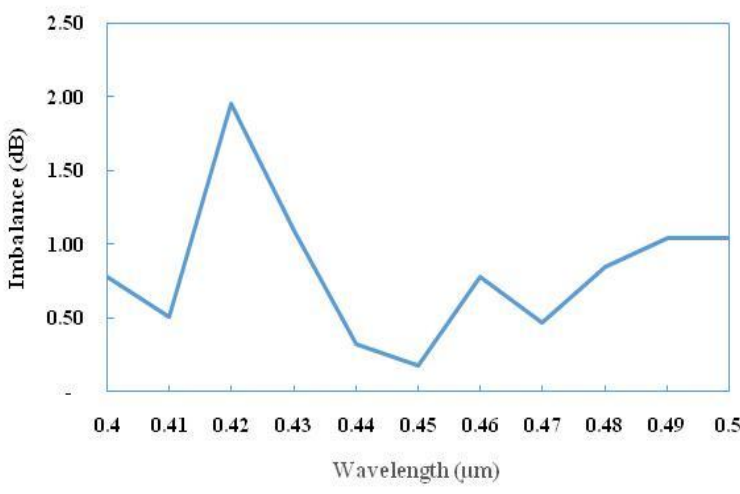

Figure 13. Power imbalance of the TE mode in the proposed design $1 \times 4$ optical power splitter 


\section{CONCLUSION}

As a conclusion, in this paper, an optical power splitter used for underwater optical communication is studied using GaN/sapphire with a short period superlattice (SPS) buffer. The numerical experimental is performed using FD-BPM and MathLab. From simulation results, it has been shown that the proposed design has an excellent performance of splitting ratio, excess loss and imbalance.

This design has the potential to be applied in underwater optical communication

\section{ACKNOWLEDGEMENTS}

This research supported by IJR Grant 2017 from RISTEK DIKTI, The Republic of Indonesia.

\section{REFERENCES}

[1] H. Kaushal and G. Kaddoum, "Underwater Optical Wireless Communication", IEEEAcess Multidisiplinary, 4, pp. 1518-1547, 2016.

[2] M. Biagi, .et al., "On Rethinking Cognitive Access for Underwater Acoustic Communications", IEEE Journal of Oceanic Engineering, vol. 41, no. 4, 2016.

[3] Y. Y. Al-Aboosi, et al., "Diurnal Variability of Underwater Acoustic Noise Characteristics in Shallow Water", TELKOMNIKA (Telecommunication, Computing, Electronics and Control), vol. 15, no. 1, pp. 314-321, 2017.

[4] Raymond C. Smith and Karen S. Baker, "Optical Properties of the Clearest Natural Waters (200-800 nm)", Applied Optics, vol. 20, no. 2, pp. 177-184, 1981.

[5] Y. C. Chi, et al., "450-nm GaN Laser Diode Enables High-Speed Visible Light Communication with 9-Gbps QAMOFDM", Optics Express, vol. 23, no. 10, 2015

[6] H.M. Oubei, et al., "14.8 Gbit/s 16-QAM-OFDM Transmission Basedon Compact 450-nm Laser for underwater Wireless Optical Communication", Optics Express, vol. 23, no. 18, 2015.

[7] P.Tian, et al., "High-speed underwater Optical Wireless Communication using a blue GaN-based Micro-LED", Optics Express, vol. 25, no. 2, pp. 1193-1201, 2017.

[8] Y. Chen, et al., "26 m/5.5 Gbps Air-water Optical Wireless Communication based on an OFDM-Modulated 520-nm Laser Diode", Optics Express, vol. 25, no. 13, pp. 14760-14765, 2017.

[9] H. Zhang, et al., "Performance Analysis of Different Modulation Techniques for Free-Space Optical Communication System", TELKOMNIKA (Telecommunication, Computing, Electronics and Control), vol. 13, no. 3, pp. 880-888, 2015.

[10] D. Li, et al., "Stimulated Emission in GaN-based Laser Diodes far below the Threshold Region", Optics Express, vol. 22, no. 3, pp. 2536-2544, 2014.

[11] R. H. Horng, et al., "High Performance GaN-based Flip-chip LEDs with different Electrode Patterns", Optics Express, vol. 22, no. S3, pp. A941-A946, 2014.

[12] R. Velazquez, et al., "Fabrications and Application of single Crystalline GaN for High-performance deep UV Photodetectors", AIP Advances 6, 085117, 2016.

[13] B. B. B. Zaken, et al., "An 8-channel Wavelength MMI Demultiplexer in slot Waveguide Structures”, Materials, vol. 9 , no. 11, p. $881,2016$.

[14] D. Malka, et al., "Design of a $1 \times 4$ Silicon-alumina Wavelength Demultiplexer based on Multimode Interference in Slot Waveguide Structures", J. Opt. 17, 125702, 2015.

[15] Saktioto, et al., "Simplified Linear Configuration Model of 3x3 Single Mode Fiber Coupler using Matrix Transfer", TELKOMNIKA (Telecommunication, Computing, Electronics and Control), vol. 13, no. 3, pp. 836-843, 2015

[16] K. S. Kim, et al., "Compact Silicon Slot Waveguide Intersection Based on Mode Transformationand Multimode Interference", IEEE Photonics Journal, vol. 6, no. 9, 4502910, 2017.

[17] N. Pendam and C. P. Vardhani, "Design, Simulation \& Optimization of 3D Low-loss Asymmetrical Y-branch Optical Power Splitter on SOI Platform", IEEE International Conference on Electrial, Electronics, and Optimization Techniques, Chennai, India, 3-5 March 2016.

[18] O. Katz and D. Malka, "Design of Novel SOI $1 \times 4$ Optical Power Splitter using seven Horizontally Slotted Waveguides", Photonics and Nanostructures - Fundamentals and Applications, vol. 25, pp. 9-13, 2017.

[19] S. C. Jain, et al., "III-Nitrides: Growth, Characterization, and Properties", Journal of Applied Physics, 87, pp. 965, 2000.

[20] A. Stolz, et al., "Optical Waveguide Loss Minimized into Gallium Nitride based Structures Grown by Metal Organic Vapor Phase Epitaxy", Applied Physics Letter, 98, 161903, 2011.

[21] R. W. Purnamaningsih, et al., "Design of GaN-based Low Loss Y-Branch Power Splitter", Makara Journal of Technology, vol. 18, no. 3, pp. 101-106, 2015.

[22] A. Arviza and R.W. Purnamaningsih, "Design of S-Bend Y-Branch Power Splitter with MMI Structure", 2014Proceeding of SPIE, International Seminar on Photonics, Optics, and Its Application, pp. 9444111-9444115, 2014.

[23] T. Sekiya, et al., "Design, Fabrication, and Optical Characteristics of Freestanding GaN Waveguides on Silicon Substrate", Journal of Vacuum Science \& Technology B, Nanotechnology and Microelectronics: Materials, Processing, Measurement, and Phenomena, 33, 031207, 2015.

[24] X. Gao, et al., "Monolithic III-Nitride Photonic Integration toward Multifunctional Devices", Optics Letters, Vol. 42, no. 23, pp. 4853-4856, 2017. 
[25] L.B.Soldano, et al., "Planar Mono-mode Optical Couplers based on Multi-mode Interference Effects", Journal of Lightwave Technology, vol. 30, no. 7, pp. 1843-1850, 1992.

[26] R. Ulrich, and G. Adele, G., "Self-imaging in Homogeneous Planar Optical Waveguides", Applied Physics Letters, vol. 27, no. 6, pp. 337-339, 1975.

[27] L. B Soldano and E. C. M. Pennings, "Optical Multi-mode Interference Devices based on Self-imaging: Principles and Applications", J. Lightwave Technology, vol. 13, no. 4, pp. 615-627, 1995.

\section{BIOGRAPHIES OF AUTHORS}

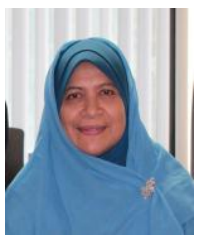

Retno Wigajatri Purnamaningsih received the undergraduate degree in Engineering Physics from the Bandung Institute of Technology, Bandung, Indonesia in 1985, the M.Eng.degree in Opto Electrotechniques and Laser Application, the Univeritas Indonesia, Jakarta, Indonesia in 1992 and the $\mathrm{Ph} . \mathrm{D}$. degree in Opto Electrotechniques and Laser Application, the Univeritas Indonesia, Jakarta, Indonesia in 2006. She is currently Assoc. Professor in Electrical Engineering Department of Universitas Indonesia. Her current research dedicates to the advancements of III-nitridewide bandgap semiconductor physics and design technologies for communication application.

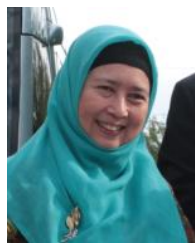

Nyi Raden Poespawati received the undergraduate degree in Electrical Engineering from the Universitas Indonesia, Depok, Indonesia in 1985, theM.Eng.degree in Electrical Engineering, the Univeritas Indonesia, Jakarta, Indonesia in 1992 and the Ph.D. degree also in Electrical Engineering, the Univeritas Indonesia, Jakarta, Indonesia in 2005. She is currently is a Full. Professor in Electrical Engineering Department of Universitas Indonesia. Her current research dedicates to the advancements of Optoelectronic Design, including III-nitride wide bandgap semiconductor physics and design technologies for communication and also solar cell.

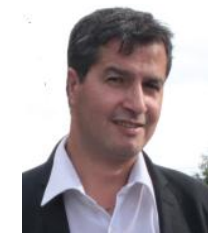

Elhadj Doghece received the PhD degree from the University of Science \& Technology in 1993 at Lille 1 - Institut d'Electronique de Microélectronique et de Nanotechnologie (IEMN), Lille, France, Lille, France. He iscurrently is a Full Professor in University of Valenciennes and Hainaud Cambresis,Valenciennes.His primary research interest are the study of new family of materials and application into future generation of active photonic devices. He has delivered numerous invited presentations in national and international conferences, and served as a review panelist for many national and international research programs. He has published over 100 refereed journal papers. 